\title{
An Unusual Cystic Sellar-Suprasellar Mass in a Young Female
}

\author{
Cristina V. Jaring and Frances Lina C. Lantion-Ang \\ Section of Endocrinology, Diabetes and Metabolism, Department of Medicine, \\ College of Medicine and Philippine General Hospital, University of the Philippines Manila
}

This is the magnetic resonance image (MRI) of the brain of a 23-year-old female with a 20-month history of headache, progressive blurring of vision, somnolence, constipation, cold intolerance and amenorrhea. Physical examination showed bitemporal hemianopsia and decreased muscle stretch reflexes for all extremities. She had normal serum sodium and urine specific gravity, modest elevation of prolactin level, low thyroxine level with inappropriately normal thyrotropin (secondary hypothyroidism), and markedly decreased 8 AM serum cortisol. The MRI of the head with gadolinium showed a moderate-sized peripherally enhancing sellar and suprasellar cystic mass measuring $3.3 \times 3.6 \times 4.0$ centimeters which is partly hemorrhagic or containing proteinaceous material, that causes marked compression of the overlying optic apparatus. Imaging findings are most compatible with a craniopharyngioma.

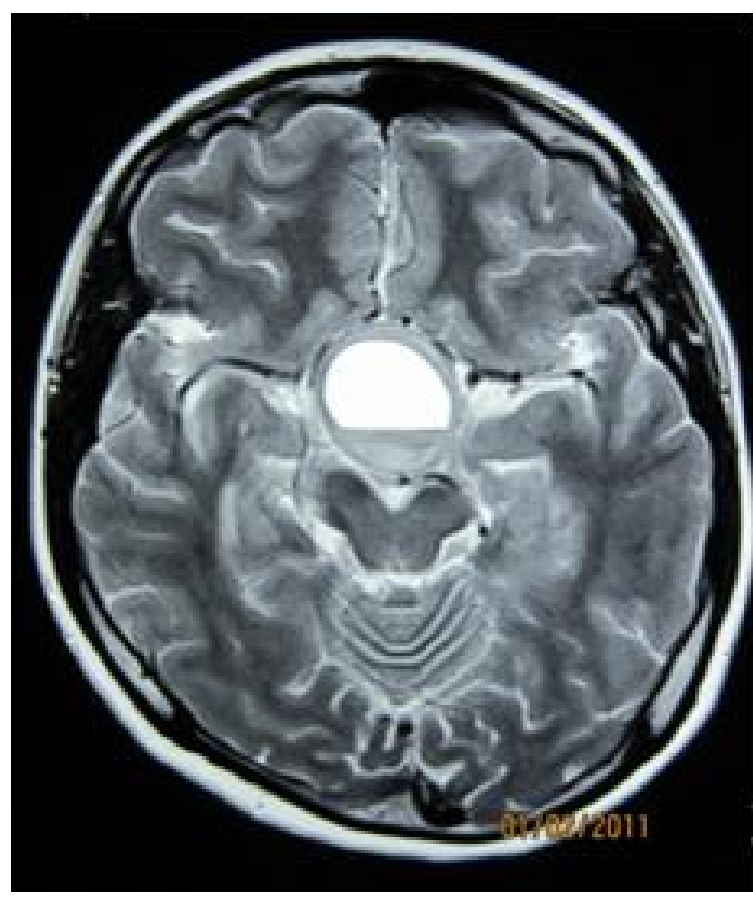

The clinical impression for this case was a sellarsuprasellar mass, probably craniopharyngioma versus pituitary macroadenoma, with optic chiasm compression and hypopituitarism (Secondary Hypothyroidism, Secondary Adrenal Insufficiency).

Craniopharyngioma is an epithelial neoplasm arising from squamous epithelial rests of the Rathke's pouch and it is the most heterogenous of the lesions involving the sellar region due to their cystic and solid components. It is usually slow-growing and symptoms frequently develop insidiously with most cases becoming obvious only after the tumor has attained a diameter of about $3 \mathrm{~cm}$. The time interval between onset of symptoms and diagnosis ranges from 1-2 years. The most common presenting symptoms are headache due to increased intracranial pressure, endocrine dysfunction resulting from mass compression of

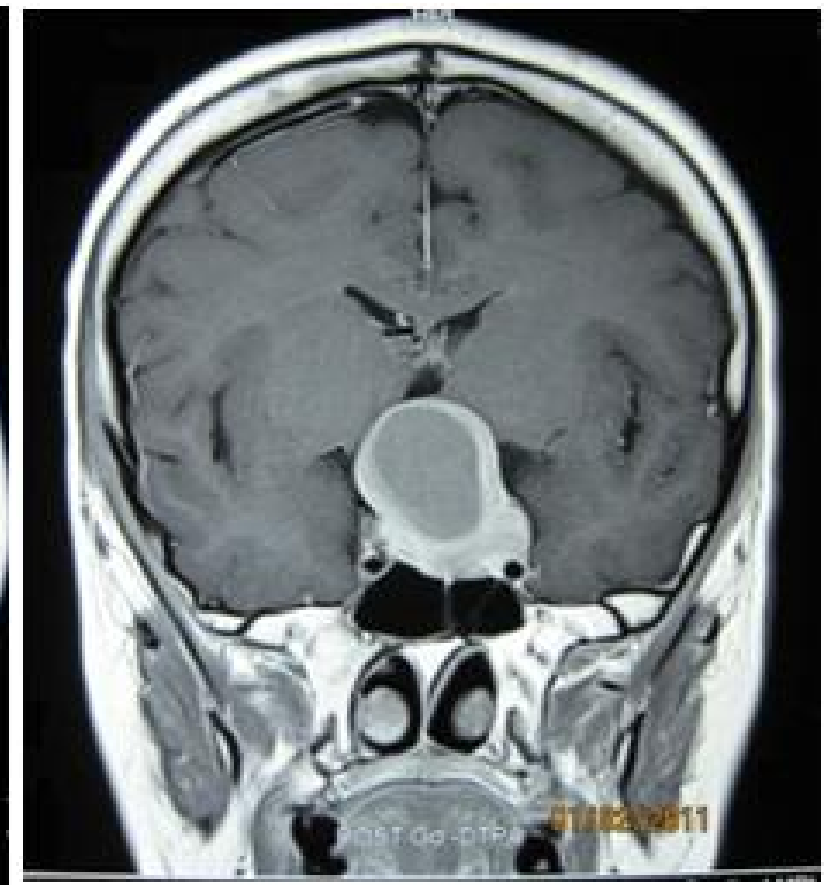

Figure 1. Sellar mass exhibiting pre-existing internal T1 hyperintensity and evidence of sedimentation leveling.

Figure 2. The sellar mass is asymmetric towards the left, extending to the left cavernous sinus region. It causes mild to moderate expansion of the floor of the sella.

ISSN 0857-1074

Copyright (C) 2011 by the JAFES

Received February 27, 2011. Accepted April 19, 2011.
Corresponding author: Cristina V. Jaring, $M D$

Section of Endocrinology, Diabetes and Metabolism, Department of Medicine, College of Medicine and Philippine General Hospital University of the Philippines Manila

Tel: 09178659771, 09228448180

E-mail:cvj521@yahoo.com 
the pituitary gland, and visual disturbances from mass compression of the optic chiasm.

The differential diagnosis for tumors in the selarsuprasellar region usually includes pituitary adenoma, craniopharyngioma, and Rathke's cleft cyst. Craniopharyngioma was mainly considered because of its characteristic appearance on MRI that was cystic but with sedimentation leveling signifying that the mass is not purely fluid but has a mixed solid and cystic content. Other MRI features suggestive of craniopharyngioma include its lobulated shape, third ventricle compression by superior tumor extension, and reticular enhancement of the solid portion. In contrast, pituitary adenomas usually have a snowman shape, solid characteristics, and homogenous enhancement of the solid portion. An ovoid shape, small tumor volume, cystic characteristics, and no or thin cyst wall enhancement are noted to be more common in Rathke's cleft cysts.

\section{References}

1. Choi SH, Kwon BJ, Na DG, et al. Pituitary adenoma, craniopharyngioma, and Rathke cleft cyst involving both intrasellar and suprasellar regions: Differentiation using MRI. Cllinical Radiology 2007; 62: 4553-462.

2. Bobustuc GC, Groves MD, Fuller GN, et al. Craniopharyngioma. http://emedicine.medscape.com/article/1157758.

3. Jameson L, De Groot L. Endocrinology-Adult and Pediatric, $6^{\text {th }}$ ed. Saunders 2010.

4. Kronenberg HM, Melmed S, Polonsky KS, Larsen PR. Williams Textbook of Endocrinology, 11 $11^{\text {th }}$ ed. Saunders 2008. 